\title{
REFLECTIONS
}

\section{Building on the first decade of ART}

G Maartens, MB ChB, FCP (SA), DTM\&H, MMed; E Goemaere, MD, DTM\&H, PhD

Corresponding author: G Maartens (gary.maartens@uct.ac.za)

Professor Gary Maartens hails from the Division of Clinical Pharmacology, Department of Medicine, University of Cape Town (UCT), South Africa. Dr Eric Goemaere is affiliated with Médecins Sans Frontières, South Africa, and the School of Public Health and Family Medicine, UCT.

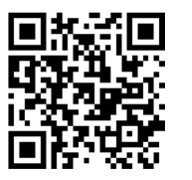

There is much to celebrate at the end of the first decade of South Africa's public sector antiretroviral therapy (ART) programme. An estimated 2 million South Africans had started ART by 2012, making ours the largest ART programme globally. ART coverage in adults, according to current guidelines, was estimated at $81 \%$ in 2012. ${ }^{[1]}$ The prevalence of HIV is increasing, because people receiving ART are living longer ${ }^{[2]}$ In rural KwaZulu-Natal, adult life expectancy increased from 49.2 years in 2003, just before the beginning of the ART programme, to 60.5 years in $2011 .^{[3]}$ Tremendous strides have been made in the prevention of mother-to-child transmission (PMTCT) of the virus. Almost $90 \%$ of pregnant, HIV-infected women access antiretrovirals (ARVs) either for their own health or for PMTCT, resulting in a 67\% decline in new infections in children from 2009 to 2012. ${ }^{[4]}$ Further declines in new infections in children should be seen with the new PMTCT guidelines, which include prolonged ARVs for infants during breastfeeding, and combination ART for all mothers irrespective of $\mathrm{CD} 4^{+}$counts. ART access in eligible children has increased from $17 \%$ in 2009 to $67 \%$ in $2012 .{ }^{[4]}$ We have even started a third-line ART programme.

The birth of our ART programme was difficult, to say the least. We should never forget the tragic loss of hundreds of thousands of South African lives as a result of delays in starting our ART programme. The AIDS-denialist views of former President Mbeki, including the absurd notion that ARVs, not HIV, were the cause of mortality, and the ill-informed promotion of nutrition as treatment for HIV by then health minister, Manto TshabalalaMsimang, were bitter pills to swallow for people living with HIV, grieving relatives, and healthcare workers. Civil society, notably the Treatment Action Campaign (TAC) and the AIDS Law Project, played a major role in forcing the government to implement ARVs for PMTCT, and later an ART programme. However, once the green light was reluctantly given to scale-up ART, the public sector responded well.

Donors contributed significantly to the setting up and running of our ART programme, but unlike other African countries (except for Botswana), our ART programme is largely funded out of the national budget. The US President's Emergency Plan for AIDS Relief and the Global Fund to Fight AIDS, Tuberculosis and Malaria have played key roles in funding our ART programme and providing technical assistance. Less well acknowledged is the critical role that donor-funded pilot ART projects have played in establishing the feasibility of public health ART programmes. Particularly noteworthy was the Khayelitsha project, which was jointly funded by Médecins Sans Frontières and the Western Cape government. Three ART clinics were opened in 2000 despite opposition from national government. Generic ARVs were imported from Brazil in 2001, provoking a statement from President Mbeki's spokesman, Smuts Ngonyama, that this was tantamount to biological warfare. The high rates of virological suppression and retention in care of the first two years of the Khayelitsha ART project ${ }^{[5]}$ were influential in the initial scaleup of ART nationally and internationally.

\section{'The AIDS-denialist views of former President Mbeki, including the absurd notion that antiretrovirals, not HIV, were the cause of mortality, and the ill-informed promotion of nutrition as treatment for HIV by then health minister, Manto Tshabalala-Msimang, were bitter pills to swallow ...'}

A reduction in ARV costs has been one of the biggest achievements of the last decade. South Africa is a major global market player given the size of its national ART programme, and the Department of Health has been able to negotiate lower drug prices, to the benefit of other low- and middle-income countries. In 2000, ART cost around US\$10 000 per year, while the currently used fixed-dose combination single tablet for first-line ART costs only US $\$ 129$ per year.

South Africa has the world's highest number of people living with HIV, estimated to be 6.1 million in 2012, ${ }^{[1]}$ nearly all of whom will require ART in the next decade. The estimated number of new HIV infections in South Africa decreased from 640000 in 2001 to 370000 in 2012, which is gratifying, but most people infected in the next decade will also need ART. Major challenges lie ahead to achieve the expansion of the ART programme, particularly if South Africa adopts the new World Health Organization (WHO) ART initiation criterion of a $\mathrm{CD} 4{ }^{+}$count $<500$ cells/ $\mu$ l. Task-shifting, such as nurse-initiated management of ART (NIMART), has increased access to ART, 
but there is a need to train more nurses. Innovative models of patient care, such as adherence clubs, ${ }^{[6]}$ should be developed, adapted to local contexts, and rolled out.

\section{'We know that over 2 million South Africans have started ART, but how many are still in care? ... the massive ART programme expansion needed in the next decade will be accompanied by high attrition rates.'}

The biggest challenge for scaling up ART for the next decade will be retention in care. We know that over 2 million South Africans have started ART, but how many are still in care? Loss to follow-up increased with time and increasing clinic population size in a large South African ART programme, ${ }^{[7]}$ suggesting that the massive ART programme expansion needed in the next decade will be accompanied by high attrition rates. Measures of retention in care at the facility level need to be collected routinely. Electronic pharmacy refills are increasingly being used and could easily identify people who are defaulting. Retention in care and rates of virological suppression according to years receiving ART should be used to identify poorly functioning clinics and regions.

The initial role for patient activist groups such as the TAC was crucial, given the state opposition to rational treatment for people living with HIV. Some might have thought that the job of activists was done once the national ART programme was launched and the current progressive Minister of Health was appointed. Unfortunately, the general lack of accountability in the public health services, as demonstrated by ongoing drug stock-outs, demonstrates the need for independent civil society groups to monitor service delivery, and where necessary, exert pressure on the health services to deliver their mandate.

Finally, more resources need to be made available for operational research to support the ART programme. South African HIV researchers have been very productive, but almost all of their major achievements have been completed using resources from international grant agencies. Budgets were made available for research to support the national HIV programmes in the mid-2000s, but this was not sustained. The Medical Research Council has made good progress in leveraging extra funding for clinical research, indicating that political will exists to support more resources for research.

\section{References}

1. UNAIDS. Report on the Global AIDS Epidemic 2013. http://www.unaids.org/en/ media/unaids/contentassets/documents/epidemiology/2013/gr2013/UNAIDS_ Global_Report_2013_en.pdf (accessed 12 January 2014).

2. Zaidi J, Grapsa E, Tanser F, Newell ML, Barnighausen T. Dramatic increases in HIV prevalence after scale-up of antiretroviral treatment: A longitudinal populationbased HIV surveillance study in rural KwaZulu-Natal. AIDS 2013;27(14):23012305. [http://dx.doi.org/10.1097/QAD.0b013e328362e832]

3. Bor J, Herbst AJ, Newell ML, Barnighausen T. Increases in adult life expectancy in rural South Africa: Valuing the scale-up of HIV treatment. Science 2013;339(6122):961-965. [http://dx.doi.org/10.1126/science.1230413]

4. UNAIDS. 2013 Progres report on the Gloval Plan Towards the Elimination of New HIV Infections Among Children by 2015 and Keeping their Mothers Alive. http://www. unaids.orgenmediaunaidscontentassetsdocumentsunaidspublication201320130625_ progress_global_plan_en.pdf (accessed 12 January 2014).

5. Coetzee D, Hildebrand K, Boulle A, et al. Outcomes after two years of providing antiretroviral treatment in Khayelitsha, South Africa. AIDS 2004;18(6):887-895.

6. Luque-Fernandez MA, Van Cutsem G, Goemaere E, et al. Effectiveness of patient adherence groups as a model of care for stable patients on antiretroviral therapy in Khayelitsha, Cape Town, South Africa. PLoS ONE 2013;8(2):e56088. [http://dx.doi. org/10.1371/journal.pone.0056088]

7. Boulle A, Van Cutsem G, Hilderbrand K, et al. Seven-year experience of a primary care antiretroviral treatment programme in Khayelitsha, South Africa. AIDS 2010;24(4):563-572. [http://dx.doi.org/10.1097/QAD.0b013e328333bfb7]

S Afr J HIV Med 2014;15(1):7-8. DOI:10.7196/SAJHIVMED.1031 